\title{
Optical Fibre Magnetic Field Sensors for Monitoring of the State of Work of Electric Motors
}

\author{
K. BArCZAK ${ }^{a, *}$, T. Pustelny ${ }^{a}, Z^{2} . Z_{\text {YCKI }}^{b}$ And T. BlazejCZyK ${ }^{b}$ \\ ${ }^{a}$ Department of Optoelectronics, Institute of Physics, Silesian University of Technology \\ Krzywoustego 2, 44-100 Gliwice, Poland \\ ${ }^{b}$ Electrotechnical Institute, Pozaryskiego 28, 04-703 Warsaw, Poland
}

\begin{abstract}
The present paper deals with investigations concerning optical magnetic sensors. The most important advantage of sensors of this kind is their safety of service and insulating power. The insensitiveness of optical sensors to electromagnetic interference was discussed and experimentally investigated. The experimental investigations were concentrated on the adequate sensing fibre as well as on the sensor based on the bulk glass cylinder. The paper presents the results of magnetooptic tests of the elaborated magnetic field optical sensors and conclusions arise from them.
\end{abstract}

PACS numbers: 42.70.-a, 42.79.Hp, 42.81.-i, 42.79.Pw, 78.20.Ls

\section{Introduction}

Direct-current motors with permanent magnets run on a risk of serious failures resulting, for example, from current shorting or emergency overloads. Such failures usually cause partial demagnetization of the magnets, which leads to a loss of a power and an efficiency of the direct-current motor. To prevent these problems, magnets which lost magnetic properties, have to be magnetized immediately during work of the electric motor. It may be realized with additional set of coils installed near the magnets. During demagnetization process, the coils can magnetize the magnets by short-time current pulses (with duration of some tens of milliseconds) [1-3]. One of the important elements of this magnetization system is a magnetic field sensor, which continually controls a state of magnetization of the permanent magnets.

Measurements of the magnetic field may be realized by means of the Hall generator (so-called - hallotron) [3-6]. However, the application of hallotrons is often troublesome because electric motors generate disturbing magnetic field which affects operation of the sensor.

The Department of Optoelectronics at the Silesian University of Technology in Gliwice, Poland and the Electrotechnical Institute in Warsaw have been cooperating for elaboration of a measuring system of magnetic field inside direct-current motors based on a magnetooptic effect [6-14]. Such kind of system consists of an optical sensor, in which changes of a state of polarization (SOP) of an electromagnetic wave result from changes of the

* corresponding author; e-mail: kamil.barczak@polsl.pl intensity of magnetic field. An electromagnetic wave is transmitted to the optical sensor and next directed to a photodetector with using optical fibres. Application of the optical fibres in the transmission path guarantees the insensibility of the measuring signal on various random and accidental electromagnetic disturbances [15-17].

\section{The magnetooptic sensor based on a fibre sensing element}

At the first stage of investigation, the magnetic-field intensity sensor with a single mode optical fibre as a sensing element was elaborated. For this system the sensing fibre was ended with an optical waveguide reflecting mirror (Fig. 1).

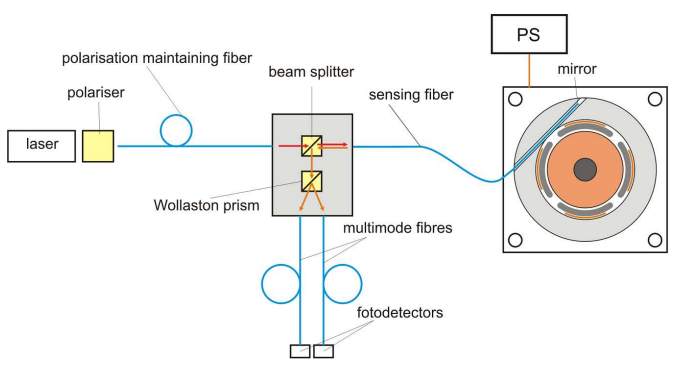

Fig. 1. Scheme of the magnetic field measuring system based on the optical fiber sensor.

In optical path a polarization maintaining (PM) fibre, a polarizer, a beam splitter, a Wollaston prism, two multimode fibres and two photodetectors were used. Monochromatic laser light after its polarization was introduced into the single mode PM fibre and next was 
directed into the sensing (sensitive to a magnetic field) fibre via a beam splitter. A part of the fibre was introduced into the magnetic field produced by the permanent magnets of the direct-current motor. After the reflection, the light was directed to the Wollaston prism where it was divided in two orthogonally polarized light beams. Both beams were coupled into the multimode fibres and transmitted to the photodetectors. Each photodetector signal was proportional to a respective polarization component of incident light and depended on the intensity of magnetic field of the magnet. Correct action of this system required to apply the beam splitter insensitive to the incident-light polarization state.

For the preliminary experiment, the Wollaston prism was replaced with the polarization analyzer yielding more complete information about the polarization state of light (Fig. 2). In these measurements an air coil was used as a controllable source of the magnetic field with the sensing fiber introduced inside the solenoid. In the investigations two type of single-mode optical fibres with cutoff wavelength at $\lambda_{1}=635 \mathrm{~nm}$ and $\lambda_{2}=1550 \mathrm{~nm}$ were used.

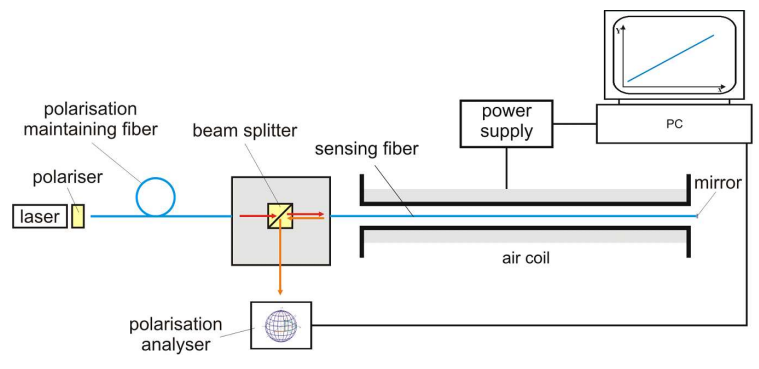

Fig. 2. Idea of the measuring setup using an optical fibre as sensing element.

\section{The magnetooptic sensor based on a bulk glass element}

Each kind of sensor, also a magnetic field sensor, ought to be insensitive to external and random changes of measuring conditions other than the changes of a quantity they measure. In this context, one of the most important problems of polarimetric sensors, which employ optical fibres, is to reduce an influence observed in them impact of random mechanical stresses on the fibers affecting the state of polarization of light propagating through the fiber $[3,13,14]$.

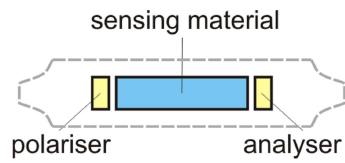

Fig. 3. Idea of the magnetic field sensor based on the glass cylinder.
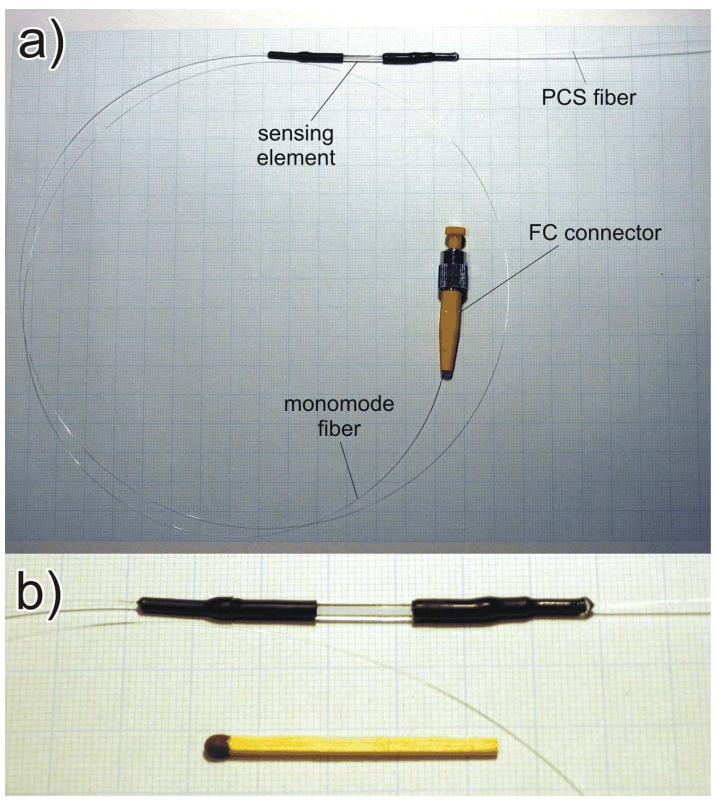

Fig. 4. Magnetic field sensor based on the glass cylinder.

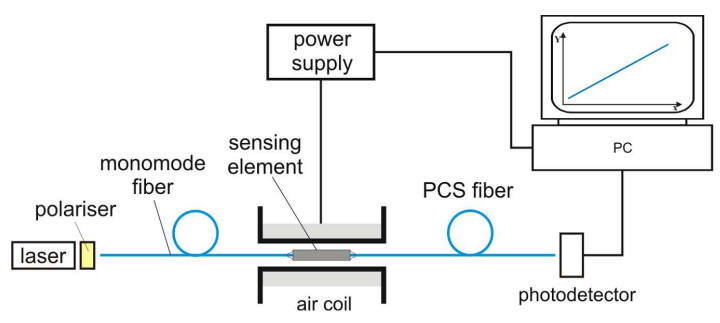

Fig. 5. Scheme for investigation of the glass sensor.

To limit the effect of mechanical stresses on the polarization state, a magnetic-field sensor in a form of a bulk-glass small cylinder was designed. In this realization the optical fibre is only applied for transmission of the optical signals and was not applied for magnetic field sensing. The glass cylinder was made of a special multicomponent glass with a diameter of $\approx 1.8 \mathrm{~mm}$ and length of $\approx 31 \mathrm{~mm}$. For this material we measured refractive index $n=1.65$ and the Verdet constant $V=4.34 \mathrm{rad} /(\mathrm{T} \mathrm{m})$ both at $\lambda=635 \mathrm{~nm}$. Two polarizers with optical fibers attached were located at the ends of the sensor (Figs. 3 and 4). A single mode optical fiber was applied at the one end of the sensing structure enabling introduction of the light into the material, while plastic-clad silica (PCS) fibre with high value of a numerical aperture $\mathrm{NA} \approx 0.30$ was used at the output end. The sensor was tested in the similar measurement configuration as earlier (Sect. 3). As an optical receiver the usual silicon photodetector was used because the analyses of polarization states were realized in the glass sensing element (the output polarizer performs a role of the analyzer of the state of polarization of the output light). Thanks to this, the measurement 
system stays to be much more simple and comfortable for using in industrial practice (Fig. 5).

\section{Results of measurements}

For the magnetic sensor based on the special optical fiber (Fig. 2) the obtained results were satisfactory only for the light wavelength $\lambda=635 \mathrm{~nm}$. The preliminary results are presented in Fig. 6. For the light of a wavelength $\lambda=1550 \mathrm{~nm}$ the polarization rotation within the testing magnetic field induction range $(0 \leq B \leq 30 \mathrm{mT})$ were immeasurably small. It is caused by a very strong dependence of the Verdet's constant value on wavelengths of light $V=f(\lambda)[6,9]$. (For $\lambda=1550 \mathrm{~nm}$ the Verdet constant $V$ has above two order small value than for $\lambda=635 \mathrm{~nm}$.) Additionally, in the optical fibre sensors the influence of random changes of stresses in the fibre on the measured values of the SOP was observed. It is the serious fault of the magnetic field system, presented in Fig. 1.

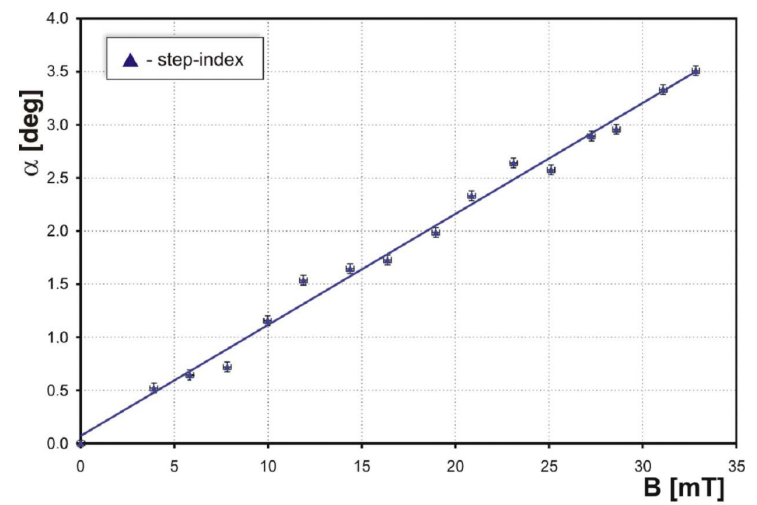

Fig. 6. Rotatory polarization as a function of external magnetic field for the sensor based on the sensing optical fibre.

Very promising results were obtained with the magnetic sensor in a form of the glass cylinder. Figure 7 presents the results of tests of this sensor (in the configuration, shown in Fig. 5). In Fig. 7 the changes of the registered signals by photodiode are presented caused by switching on and switching of the magnetic field. (By arrows the situations of switching on of the magnetic field are signed.)

\section{Conclusions}

The performed investigations showed that the magnetooptic sensors are an interesting solution for diagnosing a state of direct-current motors. In particular, they revealed that magnetooptic sensors based on glass cylinder possess some essential advantages among other sensors. For instance, the sensor based on glass cylinder has the following advantages:

— small dimensions,

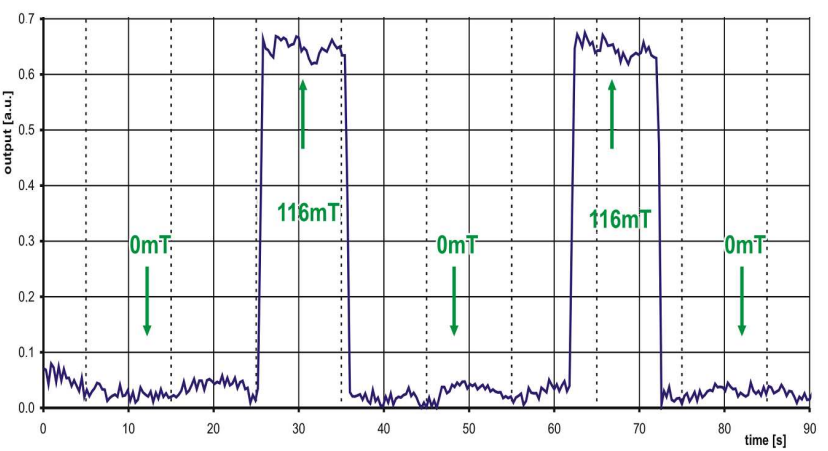

Fig. 7. Changes of signals in the glass sensor caused by external magnetic field.

— insensitivity on mechanical vibrations and bands of the optical fibres,

- easy and inexpensive means of the polarimetric signal detection,

- compact construction,

over a sensors employing the special optical fibres.

We plan to continue further investigations of bulk-glass sensors. In a near future sensors using new materials with higher Verdet's constant of $\approx 30 \mathrm{rad} /(\mathrm{T} \mathrm{m})$ will be performed. Application of this material ought to enable reduction of the dimension of the sensing element and assure the near pointed measurement of magnetic field, which is very important for some foreseen applications of the devices.

\section{Acknowledgments}

The work was sponsored by the Polish Ministry of Science and Higher Education within the grant No. N510 387735 .

\section{References}

[1] Z. Zycki, Przeglad Elektrotechniczny, 1, 97 (2007) (in Polish).

[2] T. Blazejczyk, Z. Zycki, in: VIII Sympozjum Pomiarów Magnetycznych, Politechnika Częstochowska, Częstochowa 2006, p. 13 (in Polish).

[3] T. Pustelny, Physical and Technical Aspects of Optoelectronic Sensors, Ed. SUT, Gliwice 2005, p. 86.

[4] T. Pustelny, M. Grabka, Acta Phys. Pol. A 114, A-113 (2008).

[5] Z. Zycki, T. Blazejczyk, Przeglad Elektrotechniczny, 12, 126 (2008) (in Polish).

[6] Y. Ruan, A. Ruth, A.V. Rode, S. Madden, B. Davies, Opt. Commun. 252, 39 (2005).

[7] W. Gawlik, S. Pustelny, in: New Trends in Quantum Coherence and Nonlinear Optics, Ed. R. Drampyan, Nova Sci. Publ., New York 2009, p. 57. 
[8] D. Budker, D.F. Kimball, S.M. Rochester, V.V. Yashchuk, M. Zolotorev, Phys. Rev. A 62, 043403 (2000).

[9] S. Pustelny, A. Wojciechowski, M. Gring, M. Kotyrba, J. Zachorowski, W. Gawlik, J. Appl. Phys. 103 063108 (2008).

[10] W. Gawlik, L. Krzemien, S. Pustelny, D. Sangla, J. Zachorowski, M. Graf, A.O. Sushkov, D. Budker, Appl. Phys. Lett. 88, 131108 (2006).

[11] M.P. Ledbetter, V.M. Acosta, S.M. Rochester, D. Budker, S. Pustelny, Phys. Rev. A 75, 023405 (2007).

[12] T. Pustelny, A. Opilski, B. Pustelny, Acta Phys. Pol. A 114, A-183 (2008).
[13] K. Barczak, T. Pustelny, D. Dorosz, J. Dorosz, Acta Phys. Pol. A 114, A-3 (2008).

[14] K. Barczak, T. Pustelny, D. Dorosz, J. Dorosz, Europ. Phys. J.-Special Topics 154, 11 (2008).

[15] D. Kasprzak, M. Błahut, E. Maciak, Eur. Phys. J. 154, 113 (2008).

[16] V. Balakshy, A. Vostrikova, Mol. Quant. Acoust. 28, 265 (2007).

[17] K. Gut, M. Nowak, T. Pustelny, Mol. Quant. Acoust. 28, 101 (2007). 